Atypical Antipsychotic Poisoning in Young Children:

\title{
Multicentre Analysis of Poisons Centres Data
}

3

4 Marianne Meli ${ }^{1}$, Christine Rauber-Lüthy ${ }^{1}$, Petra Hoffmann-Walbeck ${ }^{2}$, Hans-Jürgen Reinecke ${ }^{3}$, Dagmar Prasa ${ }^{4}$, Uwe 5 Stedtler ${ }^{5}$, Elke Färber ${ }^{6}$, Dieter Genser ${ }^{7}$, Hugo Kupferschmidt ${ }^{1}$, Gerd A. Kullak-Ublick ${ }^{8}$, Alessandro Ceschi ${ }^{1,8}$

Affiliations: ${ }^{1}$ Swiss Toxicological Information Centre, Associated Institute of the University of Zurich, Zurich,

8 Switzerland; ${ }^{2}$ Poison Information Centre Berlin, Berlin, Germany; ${ }^{3}$ Poison Control Centre Mainz, Mainz, Germany;

$9 \quad{ }^{4}$ Poison Information Centre Erfurt, Erfurt, Germany; ${ }^{5}$ Poison Information Centre Freiburg, Freiburg, Germany; ${ }^{6}$ Poison

10 Information Centre North, Göttingen, Germany; ${ }^{7}$ Poison Information Centre Wien, Vienna, Austria; ${ }^{8}$ Department of

11 Clinical Pharmacology and Toxicology, University Hospital Zurich, Zurich, Switzerland

12

13 Address correspondence to: Alessandro Ceschi, MD, Head, Division of Science, Swiss Toxicological Information 14 Centre, Associated Institute of the University of Zurich, Freiestrasse 16, CH-8032 Zürich, E-mail: 15 Alessandro.Ceschi@usz.ch, Tel: +41-44-634-1034, Fax: +41-44-252-8833

Keywords: Antipsychotic drugs; Drug toxicity; Poisoning; Children

Running title: Intoxication with Atypical Antipsychotics in Children

Abbreviations: bpm - beats per minute; CI - confidence interval; ECG - electrocardiogram; EPS - extrapyramidal symptoms; GCS - Glasgow Coma Scale; GfKT - Society of Clinical Toxicology; HR - heart rate; PSS - Poisoning Severity Score; QTc - QT interval corrected for heart rate; STIC - Swiss Toxicological Information Centre; WHO World Health Organization 
Abstract

28 Although paediatric patients frequently suffer from intoxications with atypical antipsychotics, the number of studies in 29 young children, which have assessed the effects of acute exposure to this class of drugs is very limited. The aim of this 30 study was to achieve a better characterization of the acute toxicity profile in young children of the atypical 31 antipsychotics clozapine, olanzapine, quetiapine, and risperidone. We performed a multicentre retrospective analysis of cases with atypical antipsychotics intoxication in children younger than 6 years, reported by physicians to German,

33 Austrian and Swiss Poisons Centres for the 9-year period between January 1, 2001 and December 31, 2009. One 34 hundred and six cases (31 clozapine, 29 olanzapine, 12 quetiapine, and 34 risperidone) were available for analysis. 35 Forty-seven children showed minor, 28 moderate, and 2 severe symptoms. Twenty-nine cases were asymptomatic. No 36 fatalities were recorded. Symptoms predominantly involved the central nervous and cardiovascular systems. Minor 37 reduction in vigilance (Glasgow Coma Scale score >9) (62\%) was the most frequently reported symptom, followed by 38 miosis (12\%) and mild tachycardia (10\%). Extrapyramidal motor symptoms were observed in one case (1\%), after 39 ingestion of risperidone. In most cases, surveillance and supportive care were sufficient to achieve a good outcome, and 40 all children made full recovery. Conclusions: Paediatric antipsychotic exposure can result in significant poisoning, 41 however in most cases only minor or moderate symptoms occurred, and were followed by complete recovery. 42 Symptomatic patients should be monitored for central nervous system depression and an electrocardiogram should be 43 obtained. 
Accidental poisoning in children represents a significant cause of paediatric morbidity [2, 5, 20]. According to the latest

Annual Report of the American Association of Poison Control Centres, children under the age of 6 years accounted for about half of all human intoxications [5], which is comparable to the situation in Switzerland (STIC Annual Report 2012). Additionally, most emergency department visits for intoxication in children involved patients younger than 6 years [30, 22]. Despite prevention efforts like introduction of child-resistant packaging and parental education, paediatric medication poisoning and emergency department visits after medication exposure is on the rise [6, 4]. Particularly the number of poisoning with antipsychotic drugs increased remarkably in recent years, with almost $8 \%$ involving children younger than 6 years [13]. Atypical antipsychotics have become first-line drugs in the treatment of schizophrenia and other common neuropsychiatric disorders, such as anxiety disorders, bipolar disorders, tic disorders and obsessive compulsive disorders, because of improved efficacy and side effect profiles, including a lower incidence of extrapyramidal side effects and tardive dyskinesia [7, 8, 12, 24]. The use of this class of drugs in children and adolescents is increasing, although frequently in an off-label or unlicensed manner $[1,12,14,16,18,21,27,35]$. They currently represent the most commonly prescribed antipsychotics for young patients [21]. Main indications in children are psychotic and several nonpsychotic conditions including attention deficit hyperactivity disorder, autism, eating disorders, tic disorders, and mental retardation associated with behavioural or psychiatric disorders [9, 23]. The increasing use made atypical antipsychotics more accessible to young children, which are in a stage of mouthing unknown objects and therefore are especially susceptible to poisoning [25]. Although antipsychotic poisoning has become a significant cause of morbidity in children, the information available in literature is limited to systematic reviews based on paediatric therapeutic studies, case reports, isolated case series, expert opinions, or extrapolations from studies in adults.

68 The purpose of this study was to achieve a better characterization of the acute toxicity profile in young children of four common atypical antipsychotics. It should help to improve the management of patients by increasing the amount of evidence to aid risk estimation of accidental ingestion.

\section{Methods}


The study was designed as a multicentre retrospective descriptive study of cases with atypical antipsychotics (clozapine, olanzapine, quetiapine (immediate and extended release) and risperidone) intoxication in children under 6 years of age. These cases were reported between January 2001 and December 2009 to poisons centres in Austria (Vienna), Germany (Berlin, Erfurt, Freiburg, Göttingen, Mainz), and Switzerland (Zurich), according to the Codex of the Society of Clinical Toxicology (GfKT) [32]. The centres were asked to provide the anonymized data in a standardized exchange 81 spreadsheet format [31]. This data, entered by physicians, included age, sex, weight, ingested drug and dose, symptoms/signs/laboratory values and causal relationship, severity of intoxication, decontamination measures, latency to decontamination, and therapeutic interventions. The first author reviewed all cases in detail before they were entered into the study to ensure that they fulfil the inclusion criteria. Doubts were resolved by consensus in an expert panel including a senior clinical toxicologist and a clinical pharmacologist with additional qualifications in general internal medicine.

87 Since not all centres classify severity of symptoms in the same way, the cases were also re-evaluated according to the Poisoning Severity Score (PSS) [28], developed by the European Association of Poison Centres and Clinical Toxicologists, the International Programme on Chemical Safety, and the European Commission.

Ethical approval was obtained from the ethics committees of the participating Poisons Centres and the study has therefore been performed in accordance with ethical standards.

Inclusion criteria

For the reported cases, the following criteria had to be fulfilled to be included in the study:

- monointoxication with either clozapine, olanzapine, quetiapine (immediate and extended release), or risperidone;

- patient age $<6$ years;

- follow-up, reported by the treating physician;

- ingested dose known (no dose range was accepted); regardless of dose, accidental ingestion of one of the drugs of interest was considered an overdose; - confirmed or likely causal relationship between exposure and clinical effect; causality assessment was based on a clear temporal relationship between drug ingestion and symptoms, absence of other drugs or diseases that can explain the symptoms, and the presence of symptoms that are described for the drug in question or are plausible from a pharmacodynamic point of view; since these criteria could not be used for asymptomatic patients, these cases were judged according to the ingested dose reported by parents or caregivers. 
109 According to the Poisoning Severity Score [28], the severity of symptoms of individual patients was classified as

110 - 'minor' if only mild, transient, and spontaneously resolving symptoms were present

111 - 'moderate' if at least one pronounced or prolonged symptom was recorded and

112 - 'severe' if at least one severe or life-threatening symptom was observed.

113 The reported symptoms and signs according to their severity are shown in Table 1.

115 Statistical Evaluation

117 The statistical analysis was performed using the software package R [29]. Descriptive statistics were used to analyse the 118 data. Correlation between age of patients and number of ingested pills was tested by Spearman rank correlation test. 119 The Wilcoxon test was used to analyse the association between gender and ingested dose, and the difference between recorded and estimated body weight. Statistical significance was defined as $\mathrm{P}<0.05$.

\section{Results}

During the study period data of 106 children fulfilling inclusion criteria were available for analysis: in 34 (32\%) cases the involved atypical antipsychotic was risperidone, in 31 (29\%) clozapine, in 29 (27\%) olanzapine, and in 12 (11\%) quetiapine (all of them immediate release tablet formulation).

Because of partially incomplete body weight data, in some cases weight had to be estimated using World Health

Organization (WHO) growth standards charts [34]. There was no significant difference between recorded and estimated weight (Wilcoxon test $\mathrm{p}=0.08)$.

Baseline characteristics of the patients are summarized in Table 2. There were 43 (41\%) females, 52 (49\%) males, and

131 in $11(10 \%)$ cases gender was not reported. The mean age of the patients was 2.6 years (range 0.8-5.5 years; median 2.3 years). No correlation between age and number of ingested pills was found (Spearman correlation coefficient, 0.16; 95\% CI, $-0.05-0.36)$.

134 The number of ingested pills ranged from 0.25 to 8, and 1 pill was ingested in almost half of intoxications (49; 46\%), 135 followed by 0.5 (15; 14\%), and 2 (13; 12\%). Only 9 (9\%) children ingested more than 2 pills. Concerning the number 136 of ingested pills, there was no significant difference between males and females (Wilcoxon test, $\mathrm{p}=0.39$ ).

137 Neurological and cardiovascular symptoms were predominating. Minor reduction in vigilance (Glasgow Coma Scale 138 score $>9)(66 ; 62 \%)$ was the most frequently reported symptom, followed by miosis $(13 ; 12 \%)$ and tachycardia 
There were 71 (67\%) presentations with an admission Glasgow Coma Scale (GCS) score of <15: in 66 (62\%) cases

141 GCS was >9 (1 GCS 11-13; 1 GCS 12; 1 GCS 13 and 63 GCS >9 with no further subdivision), in 4 (4\%) GCS was 8-9 and in $1(1 \%)$ GCS was $\leq 7$ (GCS 6). Extrapyramidal adverse effects were observed in one girl of 2.8 years after ingestion of $2 \mathrm{mg}(0.1 \mathrm{mg} / \mathrm{kg})$ of risperidone. No extrapyramidal motor symptoms were recorded after the ingestion of clozapine, olanzapine, or quetiapine. Electrocardiography was performed in 32 (30\%) children: 3 (3\%) showed extrasystoles, and in $1(1 \%)$ previously healthy girl aged 1.6 years with potassium value of $3.6 \mathrm{mmol} / \mathrm{l}$, a prolonged QTc interval (Bazett correction formula) of $468 \mathrm{msec}$ with a heart rate of $136 \mathrm{bpm}$ was recorded after the ingestion of $150 \mathrm{mg}(13.6 \mathrm{mg} / \mathrm{kg})$ of quetiapine. The observed symptoms and signs are summarized in Table 3. Gastrointestinal decontamination with activated charcoal was performed in 49 (46\%) children, in 32 (30\%) of these within 1 hour of ingestion. Gastric lavage was performed in 1 single case and 1 case of vomiting induced by parents was reported.

Overall toxicity was rated as severe in 2 (2\%), moderate in 28 (26\%), and minor in 47 (44\%) cases according to the PSS. 29 (27\%) children were asymptomatic (Table 4). No fatalities were recorded.

The toxic dose per kg body weight was estimated as the lowest dose causing objective symptoms or signs.

153 For clozapine the toxic dose was $0.8 \mathrm{mg} / \mathrm{kg}$, resulting in restlessness, ataxia, dysarthria, and somnolence; for olanzapine $1540.4 \mathrm{mg} / \mathrm{kg}$ resulting in ataxia and somnolence; for quetiapine $3.1 \mathrm{mg} / \mathrm{kg}$ resulting in ataxia and somnolence; and for risperidone $0.05 \mathrm{mg} / \mathrm{kg}$ resulting in somnolence and mild tachycardia.

The management of atypical antipsychotic toxicity mainly consisted of surveillance (64 cases; 60\%), cardiovascular and respiratory monitoring (10; 10\%), supportive care with intravenous fluids (2; $2 \%)$, potassium substitution (2; $2 \%)$, administration of oxygen (1; 1\%), administration of benzodiazepines in case of agitation or tachycardia (2; $2 \%)$, and administration of biperiden in the one case with extrapyramidal side effects $(1 ; 1 \%)$. All children showed complete recovery.

\section{Discussion}

This study investigated symptoms, signs and severity of clozapine, olanzapine, quetiapine and risperidone intoxication in children under the age of 6 years. Consistent with the literature, we found a peak incidence of intoxications in 2-yearold toddlers [5, 30, 6].

167 Neither a correlation between age and quantity of ingested pills, nor a clear imbalance between gender and occurrence of intoxication was found. Last mentioned is in contrast to previous studies, reporting a clear male predominance in childhood poisoning [5, 4].

170 Our results demonstrate that accidental poisoning with atypical antipsychotics in children under the age of 6 years, who 171 in the majority of cases ingested 0.5 to 2 pills, predominantly showed a benign clinical course with no sequelae. This 
finding is in accordance with previous reports [7, 36]. Although a significant number of patients remained asymptomatic, significant toxicity after ingestion of a single tablet of this class of drugs has been described [20,7], and susceptibility for serious toxic effects in children has been postulated $[7,10]$. However, it has to be acknowledged that the reporting of cases with severe symptoms after ingestion of low doses (i.e. publication bias) may be an issue which might lead to an overestimation of toxicity on the basis of literature data. Atypical antipsychotic intoxication in children showed the same clinical course as observed in adolescents and adults, with neurological and cardiovascular symptoms predominating [3, 7, 9, 24]. The toxic dose per kg body weight found in this study for olanzapine is similar to that described by Isbister et al. [20]. In contrast, for clozapine we found a much lower toxic dose compared to Isbister et al., which suggests a higher toxicity of this drug in overdose. To our knowledge, no toxic doses for quetiapine and risperidone have been described previously.

Central nervous system effects were the most common manifestations and ranged from somnolence, apathy and dysarthria in mild poisoning, to deep coma in severe intoxication [7]. In contrast to adults, in which central anticholinergic syndrome has been described for quetiapine, no children with such condition were observed in our study [15]. The fact that somnolence was the most common symptom is not surprising, since somnolence is already a frequently observed adverse effect in the therapeutic dose range [8, 9, 12, 27]. In accordance with previous reports, clozapine seems to be the most sedative substance $[8,27]$.

Compared with first generation antipsychotic drugs, acute extrapyramidal symptoms (EPS) are less frequent with atypical antipsychotic drugs [18, 21, 35], but seem to occur more often in children than in adults [12, 21, 17]. They typically manifest as akathisia, parkinsonism, or dystonic reactions. EPS were frequently reported after poisoning with risperidone [26], particularly in children [20, 7]. In this study, only one case of EPS resulting from risperidone poisoning was reported. No EPS were observed after exposure to clozapine, olanzapine and quetiapine, which seem to have a more favourable EPS profile [7, 8].

Cardiovascular toxicity is also an uncommon finding in atypical antipsychotic poisoning compared to intoxication with first generation antipsychotics [7, 33]. Main cardiovascular manifestations are tachycardia, hypotension and prolongation of the QT interval [33], which are largely an extension of pharmacological effects. In accordance with the literature [12, 24] and observations in the therapeutic dose range, tachycardia was the most common cardiovascular symptom in overdose. Although intoxication with atypical antipsychotics has been reported to be associated with ECG changes and also QT prolongation with the inherent risk of torsades de pointes [35, 33], only minor ECG changes were recorded in this study, with a single case of moderate prolongation of the QTc interval in a previously healthy child after exposure to quetiapine, which is probably due to overestimation of the QT by Bazett correction because of tachycardia [11]. This confirms the rarity of serious cardiovascular effects in atypical antipsychotic poisoning. 
Unfortunately, no analysis of the effect of gastrointestinal decontamination on the severity of the intoxication could be performed because of low case numbers, heterogeneity of the study population, and absence of a control group.

\section{Conclusions}

We demonstrated that the majority of children poisoned with the atypical antipsychotic drugs clozapine, olanzapine, quetiapine, and risperidone had a benign clinical course. Symptoms predominantly involved the central nervous and cardiovascular systems. Extrapyramidal side effects were rare, and the only case reported was caused by risperidone intoxication. We identified toxic doses of the four investigated substances, and this may be helpful for the clinician for risk estimation after accidental ingestion. We recommend to monitor symptomatic patients for central nervous system depression and to obtain an electrocardiogram with a focus on rhythm disturbances and ECG intervals.

\section{Study limitations}

This study has a number of limitations, which are primarily related to the retrospective nature of the study design and the relatively small sample size. Last mentioned is mainly due to our strict inclusion criteria, in particular the decision to only include monointoxications and cases with well-defined ingested doses, which we are convinced were necessary to be able to interpret the findings properly, in particular because in most cases we were not able to obtain plasma concentrations of the investigated substances to confirm the ingested amount.

Larger multicentre series of atypical antipsychotic poisoning in children have, however, not been published to date. Furthermore, it is likely that not all cases which occurred in the referral population were reported to involved poisons centres, and that bias toward reporting of the more severe cases occurred. Data are also partially incomplete, which is the nature of retrospective studies using poison centre data [19]. The use of population data to substitute for missing body weight data is a clear, but unavoidable, limitation.

Furthermore, the quantities of ingested drugs are estimates derived from the best information provided by the family member or caregiver and may over- or underestimate the actual ingestion. extended to children with larger overdoses. 
233 Funding Source

234

235 No funding was secured for this study.

236

237 Conflict of Interest

238

239 The authors declare that they have no conflict of interest.

240 
1. Almandil NB, Wong IC (2011) Review on the current use of antipsychotic drugs in children and adolescents. Arch Dis Child Educ Pract Ed 96:192-196

2. Anderson M. Poisoning in young children (2012) Arch Dis Child 97:831-832

3. Antia SX, Sholevar EH, Baron DA (2005) Overdoses and ingestions of second-generation antipsychotics in children and adolescents. J Child Adolesc Psychopharmacol 15:970-985

4. Bond GR, Woodward RW, Ho M (2012) The growing impact of pediatric pharmaceutical poisoning. J Pediatr $160: 265-270$

5. Bronstein AC, Spyker DA, Cantilena LR, Jr., Rumack BH, Dart RC (2012) 2011 Annual Report of the American Association of Poison Control Centers' National Poison Data System (NPDS): 29th Annual Report. Clin Toxicol (Phila) 50:911-1164

6. Budnitz DS, Salis S (2011) Preventing medication overdoses in young children: an opportunity for harm elimination. Pediatrics 127:1597-1599

7. Burns MJ (2001) The pharmacology and toxicology of atypical antipsychotic agents. J Toxicol Clin Toxicol $39: 1-14$

8. Caccia S, Clavenna A, Bonati M (2011) Antipsychotic drug toxicology in children. Expert Opin Drug Metab Toxicol 7:591-608

10. Catalano G, Cooper DS, Catalano MC, Butera AS (1999) Olanzapine overdose in an 18-month-old child. J Child Adolesc Psychopharmacol 9:267-271

9. Campbell M, Rapoport JL, Simpson GM (1999) Antipsychotics in children and adolescents. J Am Acad Child

13. Cobaugh DJ, Erdman AR, Booze LL, Scharman EJ, Christianson G, Manoguerra AS, Caravati EM, Chyka PA, Woolf AD, Nelson LS, Troutman WG (2007) Atypical antipsychotic medication poisoning: an evidence-based consensus guideline for out-of-hospital management. Clin Toxicol (Phila) 45:918-942

14. Dubois D (2005) Toxicology and overdose of atypical antipsychotic medications in children: does newer necessarily mean safer? Curr Opin Pediatr 17:227-233 
15. Eyer F, Pfab R, Felgenhauer N, Strubel T, Saugel B, Zilker T (2011) Clinical and analytical features of severe suicidal quetiapine overdoses--a retrospective cohort study. Clin Toxicol 49:846-853

16. Findling RL, McNamara NK (2004) Atypical antipsychotics in the treatment of children and adolescents: clinical applications. J Clin Psychiatry 65 Suppl 6:30-44

17. Findling RL, McNamara NK, Gracious BL (2000) Paediatric uses of atypical antipsychotics. Expert Opin Pharmacother 1:935-945

18. Fleischhaker C, Heiser P, Hennighausen K, Herpertz-Dahlmann B, Holtkamp K, Mehler-Wex C, Rauh R, Remschmidt H, Schulz E, Warnke A (2006) Clinical drug monitoring in child and adolescent psychiatry: side effects of atypical neuroleptics. J Child Adolesc Psychopharmacol 16:308-316

19. Hoffman RS (2007) Understanding the limitations of retrospective analyses of poison center data. Clin Toxicol (Phila) 45:943-945

20. Isbister GK, Balit CR, Kilham HA (2005) Antipsychotic poisoning in young children: a systematic review. Drug saf 28:1029-1044

21. Liebelt EL (2005) New drug therapies for diseases in children: from atypical antipsychotics to antivenom for snakebites. Curr Opin Pediatr 17:221-222

22. McCaig LF, Burt CW (1999) Poisoning-related visits to emergency departments in the United States, 19931996. J Toxicol Clin Toxicol 37:817-826

23. McKinney C, Renk K (2011) Atypical antipsychotic medications in the management of disruptive behaviors in

24. Minns AB, Clark RF (2012) Toxicology and overdose of atypical antipsychotics. J Emerg Med 43:906-913

25. Moya J, Bearer CF, Etzel RA (2004) Children's behavior and physiology and how it affects exposure to

27. Patel NC, Crismon ML, Hoagwood K, Johnsrud MT, Rascati KL, Wilson JP, Jensen PS (2005) Trends in the use of typical and atypical antipsychotics in children and adolescents. J Am Acad Child Adolesc Psychiatry 44:548-556 environmental contaminants. Pediatrics 113(4 Suppl):996-1006

26. Page CB, Calver LA, Isbister GK (2010) Risperidone overdose causes extrapyramidal effects but not cardiac toxicity. J Clin Psychopharmacol 30:387-390

29. R Core Team (2012) R: A language and environment for statistical computing. R Foundation for Statistical Computing, Vienna, Austria. ISBN 3-900051-07-0. Available via: http://www.R-project.org/ 
303 30. Schillie SF, Shehab N, Thomas KE, Budnitz DS (2009) Medication overdoses leading to emergency department visits among children. Am J Prev Med 37:181-187

305 31. Stedtler U, Hoffmann-Walbeck P, Prasa D, Rauber-Luethy C, Reinecke HJ (2008) Amlodipine: collection and analysis of case data in the society of clinical toxicology of German speaking countries (GfKT). EAPCCT Abstracts. Clin Toxicol (Phila) 46:351-421

32. Stürer A, Felgenhauer N, Hentschel H, Hruby K, Seidel C, Lampe D, Kupferschmidt H (2013) Codex über die Zusammenarbeit und die Nutzung gemeinsamer Daten der Giftinformationszentren. Available via: http://www.klinitox.de/fileadmin/DOKUMENTE/GESELLSCHAFT/GfKT_CODEX_v1.0_071108.pdf Acessed January 2013

33. Tan HH, Hoppe J, Heard K (2009) A systematic review of cardiovascular effects after atypical antipsychotic medication overdose. Am J Emerg Med 27:607-616

314 34. The WHO Child Growth Standards. Available via: http://www.who.int/childgrowth/standards Acessed January 2013

35. Vitiello B, Correll C, van Zwieten-Boot B, Zuddas A, Parellada M, Arango C (2009) Antipsychotics in children and adolescents: increasing use, evidence for efficacy and safety concerns. Eur Neuropsychopharmacol 19:629-635

36. Wong DC, Curtis LA (2004) Are 1 or 2 dangerous? Clozapine and olanzapine exposure in toddlers. J Emerg Med 27:273-277 
Table 1. Symptoms and severity of intoxication in the study population

Table 2. Patient baseline characteristics

Table 3. Observed symptoms and signs

Table 4. Severity of intoxication and ingested dose 